\title{
Development of Second-Generation VEGFR Tyrosine Kinase Inhibitors: Current Status
}

\author{
Pankaj Bhargava $\cdot$ Murray O. Robinson
}

Published online: 12 February 2011

(C) The Author(s) 2011. This article is published with open access at Springerlink.com

\begin{abstract}
The vascular endothelial growth factor (VEGF) signaling pathway appears to be the dominant pathway involved in tumor angiogenesis, providing a rationale for targeting the VEGF receptors (VEGFR-1, -2, and -3) in the treatment of cancers. In particular, VEGF signaling is thought to be important in renal cell carcinoma (RCC) because of the deregulation of the pathway through nearly uniform loss of the von Hippel Lindau protein. The tyrosine kinase inhibitors (TKIs) sorafenib, sunitinib, and pazopanib are approved by the US Food and Drug Administration for the treatment of advanced RCC; however, these multitargeted agents inhibit a wide range of kinase targets in addition to the VEGFRs, resulting in a range of adverse effects unrelated to efficient VEGF blockade. This article reviews recent advances in the development of the secondgeneration VEGFR TKIs, including the more selective VEGFR TKIs tivozanib and axitinib, and focuses on the potential benefits of novel inhibitors with improved potency and selectivity.
\end{abstract}

Keywords Angiogenesis · Axitinib · Cediranib · Growth factors · Pazopanib - Renal cell carcinoma · Sorafenib · Sunitinib - Tivozanib · Tyrosine kinase inhibitors · Vascular endothelial growth factor

\footnotetext{
P. Bhargava $\cdot$ M. O. Robinson $(\square)$

AVEO Pharmaceuticals, Inc.,

75 Sidney Street, 4th floor,

Cambridge, MA 02139, USA

e-mail: mrobinson@aveopharma.com

P. Bhargava

e-mail: pbhargava@aveopharma.com
}

\section{Introduction}

Angiogenesis, the formation of new capillary blood vessels, is fundamental to normal development and critical for physiological processes in adults, such as reproduction and wound healing. Angiogenesis is also associated with pathologic conditions, such as rheumatoid arthritis, agerelated macular degeneration, and diabetic retinopathy, and is also a crucial component of tumor growth and metastasis [1]. As a nascent tumor grows, the cell mass limits diffusion of oxygen, creating hypoxia, which in turn activates the hypoxia-inducible factor (HIF) transcription factors and thereby upregulates expression of the vascular endothelial growth factor (VEGF) family of proteins. When combined with appropriate proteolytic factors in the microenvironment, the VEGFs enable the recruitment and proliferation of nearby vessel structures to initiate and sustain tumor neovasculature [2]. Evidence suggests that acquisition of a blood supply is a rate-limiting step in the establishment of solid tumors. Thus, inhibition of angiogenesis has emerged as an important antitumor strategy for solid tumors [3].

While a number of angiogenesis inducers have been identified [1], the VEGF signaling pathway appears to be the dominant pathway involved in tumor angiogenesis [3]. The VEGF family consists of five structurally related proteins (VEGF-A, VEGF-B, VEGF-C, VEGF-D, and placental growth factor [PLGF]), and signaling through this pathway is mediated by the binding of these growth factors to three receptors (VEGF receptor [VEGFR]-1, VEGFR-2, and VEGFR-3). The ligands bind each receptor with distinct but overlapping specificity as well as distinct biological function, together acting to affect proliferation, migration, and morphogenesis of endothelial cells to form functional vasculature $[4 \cdot, 5,6,7]$. 
Although VEGF signaling is important for the growth of many different tumor types, advances in our understanding of tumor cell biology have indicated a particularly strong rationale for blocking VEGF as a treatment strategy in clear cell renal cell carcinoma (RCC). Functional defects in the von Hippel Lindau gene (VHL), which is a negative regulator of HIF1 and HIF2 and thus a tumor suppressor, are present in over $90 \%$ of clear cell RCC tumors [8]. VHL inactivation results in the stabilization of HIFs, particularly HIF2 [9, 10], and upregulation of the expression of a large set of hypoxia-induced genes, including VEGF-A and VEGF-C [8].

Therapeutic inhibition of the VEGF pathway may be achieved via monoclonal antibodies or receptor traps targeted to the various VEGF ligands (eg, bevacizumab binding to VEGF-A, TB-403 binding to PLGF, aflibercept binding to VEGFs), antibodies targeting the extracellular domain of various VEGFRs (IMC-1C11 binding to VEGFR-2), or via intracellular inhibition of VEGF signaling through use of small-molecule tyrosine kinase inhibitors (TKIs) that target the intracellular kinase domains of the three VEGFRs [11]. This article reviews recent advances in the development of second-generation VEGFR TKIs, focusing on the potential benefits of novel inhibitors with improved potency and selectivity.

\section{Approved TKIs with Anti-VEGFR Activity}

Over the past 4 years, three oral multitargeted TKIs, sorafenib $[12 \bullet \cdot]$, sunitinib $[13 \bullet \bullet, 14]$, and pazopanib [15••], have been approved by the US Food and Drug Administration (FDA) and the European Medicines Agency for the treatment of advanced RCC. In addition to the VEGFR tyrosine kinases, these agents potently inhibit a wide range of tyrosine kinases and other targets (such as platelet-derived growth factor receptor [PDGFR], stem cell factor receptor [c-kit], FMS-like tyrosine kinase-3 [Flt3], rearranged during transfection [RET], colonystimulating factor 1 receptor [CSF1R], c-Raf, and Raf homolog B1 [B-Raf]), which disrupt multiple signaling pathways $[12 \bullet, 13 \bullet \bullet, 14,15 \bullet \bullet, 16]$. This lack of specificity for the VEGFRs is manifested in the occurrence of several toxicities that are unrelated to blockage of the VEGF pathway, often termed "off-target" effects of multitargeted TKIs. These toxicities have not been observed with the monoclonal antibody bevacizumab, which is a selective VEGF pathway inhibitor available for human use.

A phase 3 randomized study [13••] comparing oral sunitinib $(50 \mathrm{mg}$ once daily for 4 weeks, followed by 2 weeks without treatment) with subcutaneously administered interferon (IFN)- $\alpha$ (9 million units 3 times weekly) as first-line treatment in 750 patients with metastatic RCC showed significant improvement in median progression-free survival (PFS; 11 months vs 5 months, respectively; $P<$ 0.001 ) and objective response rate (ORR; $31 \%$ vs $6 \%$, respectively; $P<0.001)$ with sunitinib. While IFN- $\alpha$ was associated with a higher incidence of grade 3 or 4 treatmentrelated fatigue $(12 \%$ vs $7 \%$ with sunitinib; $P<0.05)$, sunitinib was associated with a higher incidence of grade 3 diarrhea $(5 \%$ vs $0 \%)$, vomiting ( $4 \%$ vs $1 \%)$, hypertension ( $8 \%$ vs $1 \%$ ), and hand-foot syndrome ( $5 \%$ vs $0 \% ; P<0.05$ for all comparisons). Sunitinib was also associated with a higher incidence of grade 3 or 4 neutropenia ( $12 \%$ vs $7 \%$, respectively, including two cases of febrile neutropenia with sunitinib) and thrombocytopenia ( $8 \%$ vs $0 \% ; P<0.05$ for all comparisons). A total of $38 \%$ of patients in the sunitinib group required a dose reduction, and $32 \%$ required a dose interruption.

The pivotal phase 3 , randomized, placebo-controlled study [12••] of sorafenib (TARGET) enrolled 903 patients with advanced clear cell RCC that was resistant to therapy with cytokines. Treatment with oral sorafenib $400 \mathrm{mg}$ twice daily significantly prolonged PFS (5.5 months) compared with placebo (2.8 months; $P<0.01)$; overall survival (OS) was not significantly different between the treatment groups. Partial responses were reported for $10 \%$ of sorafenib-treated patients compared with $2 \%$ in the placebo group $(P<0.001)$. The most common grade 3 or 4 adverse events with sorafenib included hand-foot skin reactions $(6 \%)$, fatigue $(5 \%)$, dyspnea $(4 \%)$, and diarrhea (2\%); grade 3 or 4 hypertension and cardiac ischemia were rare serious adverse events occurring more often with sorafenib ( $4 \%$ and $3 \%$, respectively) than with placebo $(<1 \%$ for both).

The activity of pazopanib was assessed in a randomized, placebo-controlled, phase 3 study involving 435 patients with locally advanced or metastatic RCC (46\% of whom were previously treated with cytokines) [15••]. Median PFS was significantly longer with pazopanib compared with placebo in the overall study population (9.2 months vs 4.2 months; $P<0.0001$ ), as well as in the treatment-naive (11.1 months vs 2.8 months; $P<0.0001)$ and cytokinepretreated subpopulations (7.4 months vs 4.2 months; $P<$ $0.001)$. ORR was also significantly greater with pazopanib (30\%) compared with placebo $(3 \% ; P<0.001)$. The most common grade 3 and 4 adverse events associated with pazopanib included diarrhea (4\%), hypertension (4\%), lymphocytopenia (4\%), and asthenia (3\%). Abnormalities in hepatic function were more frequent in the pazopanib arm (alanine transaminase $\geq 3$ times the upper limit of normal in $18 \%$ of patients), and were associated with two treatment-related deaths. In an expert review at the FDA Oncology Drug Advisory Committee meeting, hepatotoxicity with pazopanib was felt to be similar to that seen with sunitinib during their phase 3 trial [17]. 


\section{Selectivity and Potency of VEGFR Inhibitors}

Although multitargeted TKIs have demonstrated antitumor activity, they are associated with a variety of "off-VEGF target" effects related to their nonspecific nature [16]. For example, hand-foot skin reactions, fatigue, stomatitis, diarrhea, hair color changes, myelosuppression, and thyroid dysfunction are commonly associated with treatment with multitargeted TKIs. Low potency of currently available TKIs (Fig. 1a) requires administration of higher doses to obtain optimal VEGFR blockade and efficacy; however, higher doses are in turn associated with increased blockade of non-VEGF kinases due to low selectivity (Fig. 1b), leading to toxicities that often require dose reductions or interruptions. The offtarget effects of multitargeted TKIs have also limited their use in combination regimens due to overlapping toxicities with chemotherapeutic drugs. These limitations of multitargeted TKIs have led to the development of more selective and potent anti-VEGFR TKIs (Table 1), with the objective of providing improved antitumor activity with fewer off-target toxicities at therapeutic doses.

\section{Second-Generation VEGFR TKIs}

Tivozanib

Tivozanib (AV-951) is an extremely potent and selective oral pan-VEGFR TKI with picomolar potency to each of the three VEGFRs (VEGFR-1, $0.21 \mathrm{nM}$; VEGFR-2, 0.16 $\mathrm{nM}$; VEGFR-3, $0.24 \mathrm{nM}$ ), which results in a high selectivity for the VEGFRs relative to other kinases [18,

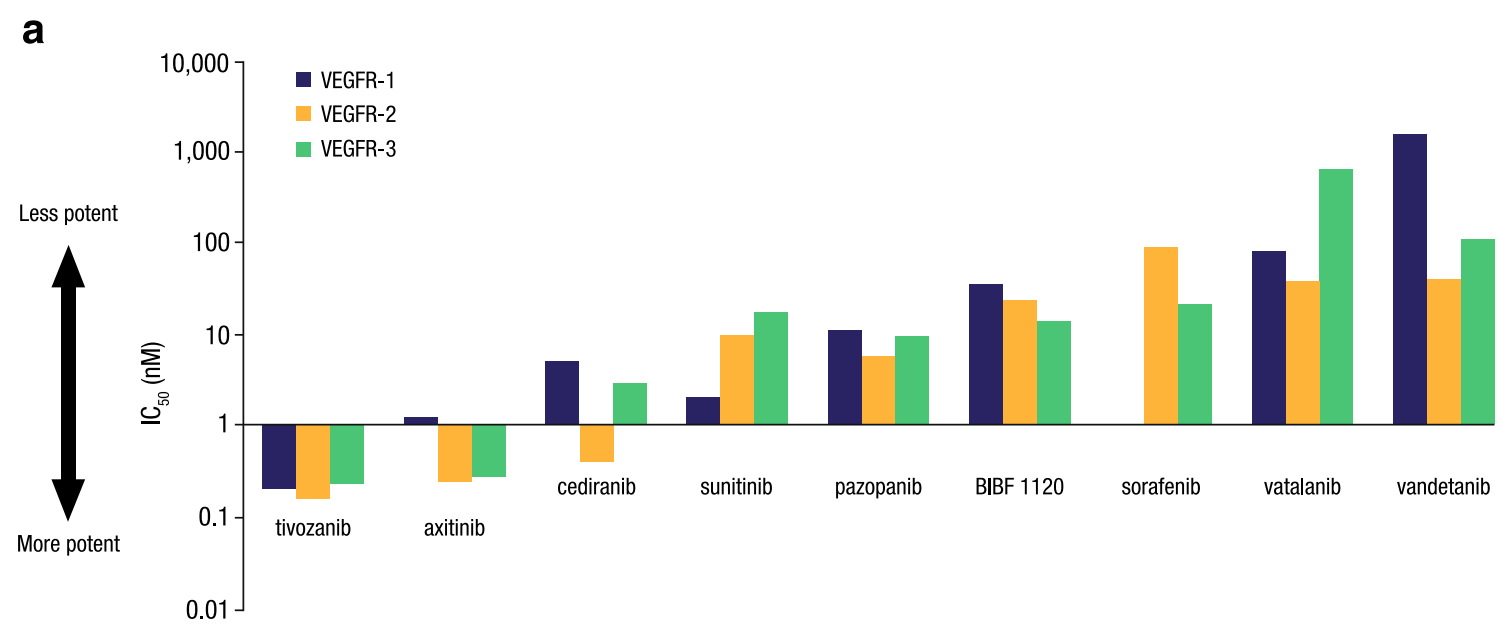

b

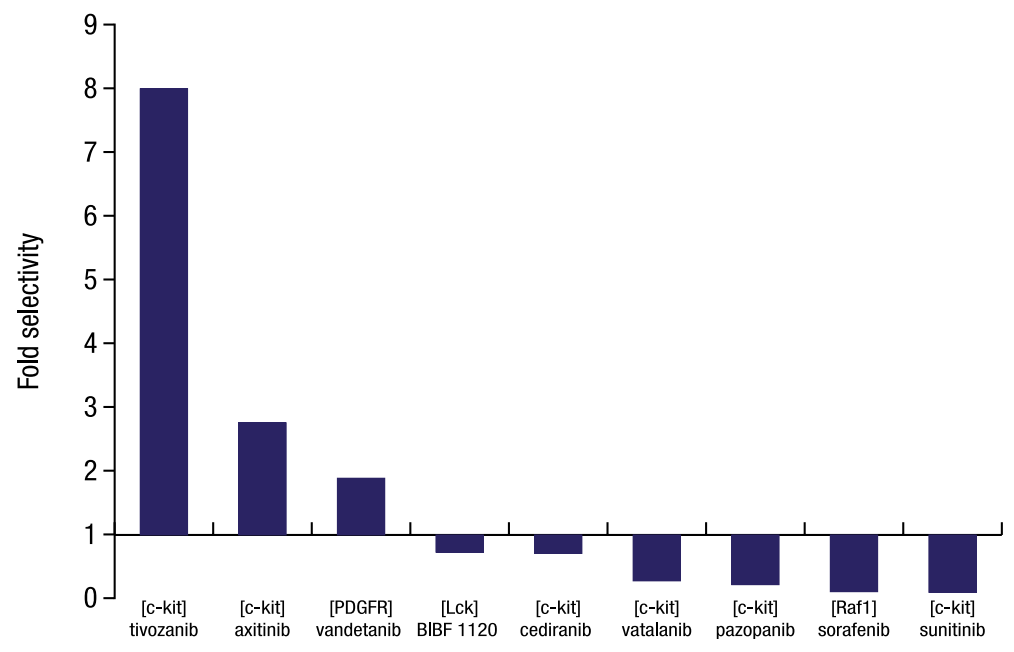

Fig. 1 Relative VEGFR potencies a and selectivities b of multitargeted and VEGFR TKIs [19, 23, 25•, 49, 64-66]. Potency toward the VEGFR-1, -2, and -3 kinases is expressed as $\mathrm{IC}_{50}$ values. Selectivity was calculated as the ration of mean potency toward VEGFR-1, -2 , and -3 versus the next most potent off-target kinase (indicated in brackets) for each agent; selectivity $<1$ indicates a higher potency for the off-target kinase versus VEGF kinase. $c$-kit stem cell factor receptor; $I C_{50}$ half-maximal inhibitory concentration; $P D G F R$ platelet-derived growth factor receptor; TKI tyrosine kinase inhibitor; $V E G F R$ vascular endothelial growth factor receptor 
Table 1 Clinical activity and tolerability of monotherapy with second-generation VEGFR TKIs in RCC

\begin{tabular}{|c|c|c|c|}
\hline Agent & Study design & Activity & Grade $\geq 3$ adverse events (active treatment arms) \\
\hline \multirow[t]{2}{*}{ Axitinib } & $\begin{array}{l}\text { Phase } 2 \text {, single-arm, } 52 \text { patients } \\
\text { with clear cell mRCC [25•] }\end{array}$ & $\begin{array}{l}\text { ORR } 44 \% \text {; TTP } 15.7 \text { months; } \\
\text { OS } 29.9 \text { months }\end{array}$ & $\begin{array}{l}\text { Hypertension }(8 \%) \text {, diarrhea }(5 \%) \text {, fatigue }(4 \%) \text {, } \\
\text { anorexia }(1 \%) \text {, limb pain }(2 \%) \text {, arthralgia }(1 \%) \text {, } \\
\text { myalgia }(1 \%) \text {, stomatitis }(1 \%)\end{array}$ \\
\hline & $\begin{array}{l}\text { Phase } 2 \text {, single-arm, } 62 \text { patients with } \\
\text { sorafenib-refractory clear cell mRCC [26•] }\end{array}$ & $\begin{array}{l}\text { ORR } 23 \% \text {; PFS } 7.4 \text { months; } \\
\text { OS } 13.6 \text { months }\end{array}$ & $\begin{array}{l}\text { Hand-foot syndrome }(16 \%) \text {, fatigue }(16 \%) \text {, } \\
\text { hypertension }(16 \%) \text {, dyspnea }(15 \%) \text {, diarrhea } \\
(15 \%) \text {, dehydration }(8 \%) \text {, hypotension }(7 \%)\end{array}$ \\
\hline \multirow[t]{3}{*}{ Tivozanib } & $\begin{array}{l}\text { Phase } 2 \text {, randomized discontinuation } \\
\text { study of } 272 \text { patients with locally } \\
\text { advanced or mRCC (all histologies) [20•] }\end{array}$ & ORR 27\%; PFS 11.8 months & Hypertension $(9 \%)$, asthenia $(2 \%)$ \\
\hline & $\begin{array}{l}\text { Randomized, placebo-controlled } \\
\text { phase }(n=111)[21 \bullet]\end{array}$ & Tivozanib: PFS 12.1 months & \\
\hline & & Placebo: PFS 6.3 months & \\
\hline \multirow[t]{2}{*}{ Cediranib } & $\begin{array}{l}\text { Phase 2, randomized, placebo- } \\
\text { controlled; } 71 \text { patients with } \\
\text { advanced RCC [37] }\end{array}$ & $\begin{array}{l}\text { Cediranib: tumor size }-20 \% \text {; } \\
\text { ORR 34\%; PFS } 12.1 \text { months }\end{array}$ & $\begin{array}{l}\text { Fatigue }(19 \%) \text {, hypertension }(19 \%), \\
\text { diarrhea }(13 \%)\end{array}$ \\
\hline & & $\begin{array}{l}\text { Placebo: tumor size }+19 \% \text {; } \\
\text { PFS } 2.7 \text { months }\end{array}$ & \\
\hline
\end{tabular}

$m R C C$ metastatic renal cell carcinoma; ORR objective response rate; TTP time to progression; $O S$ overall survival; $P F S$ progression-free survival; $R C C$ renal cell carcinoma; TKI tyrosine kinase inhibitor; VEGFR vascular endothelial growth factor receptor.

19]. A phase 2 randomized discontinuation trial [20•] involving patients with locally advanced or metastatic RCC $(83 \%$ of whom had clear cell RCC, and $73 \%$ of whom had undergone nephrectomy) evaluated 16 weeks of open-label treatment with tivozanib $1.5 \mathrm{mg} / \mathrm{day}$, after which patients who had $<25 \%$ tumor change were randomized to 12 weeks of treatment with tivozanib or placebo. Preliminary results indicate that among all treated patients $(N=272)$, tivozanib was associated with an ORR of $27 \%$ and a median PFS of 11.8 months. Among those with clear cell RCC who had undergone nephrectomy $(n=176)$, the ORR was $32 \%$ and the median PFS was 14.8 months [20•]. Among those patients randomized to double-blind treatment, median PFS was longer among those randomized to receive tivozanib ( $n=58 ; 12.1$ months) compared with placebo ( $n=53 ; 6.3$ months), with a significantly greater proportion of patients progression free after 12 weeks of treatment on the tivozanib arm $(P=0.003)[21 \bullet$. Of the 29 patients with progressive disease while on placebo, 26 patients crossed back to open-label tivozanib and 24 experienced response or stable disease. The most common adverse events observed in $>20 \%$ (any grade) were hypertension (50\%) and dysphonia (22\%); the incidences of gastrointestinal events, fatigue, and hand-foot syndrome were low. Grade 3 adverse events included hypertension $(8 \%)$ and asthenia $(2 \%)$; an additional $1 \%$ of patients experienced grade 4 hypertension [20•]. As observed with agents approved in RCC, response to tivozanib and median PFS were higher among patients experiencing hypertension during treatment compared with those that did not [22]. An ongoing open-label phase 3 trial (TIVO-1) is comparing tivozanib versus sorafenib in treatment-naive or cytokinepretreated patients with advanced clear cell RCC who have undergone a nephrectomy (ClinicalTrials.gov identifier: NCT01030783).

Preliminary results from an ongoing phase 1 study [23] evaluated the combination of tivozanib with temsirolimus, a mammalian target of rapamycin (mTOR) inhibitor, in patients with metastatic RCC. The combination was well tolerated at full doses of each agent (tivozanib $1.5 \mathrm{mg}$ /day and temsirolimus $25 \mathrm{mg} /$ week), with no dose-limiting toxicities observed and no evidence of pharmacokinetic interaction between tivozanib and temsirolimus. Clinical activity was encouraging, with 2 of 16 patients achieving confirmed partial responses and 8 patients achieving stable disease for $>10$ weeks as of the cutoff date. Grade 3 adverse events included thrombocytopenia, fatigue/asthenia, hypertension, and rash, each of which was reported by 1 patient $(6 \%)$ [23]. Tivozanib is the first TKI to be safely combined with an mTOR inhibitor at full dose and schedule of both agents. A study evaluating the combination of tivozanib with everolimus is ongoing (ClinicalTrials.gov identifier: NCT01058655).

Tivozanib is also currently being evaluated in patients with other cancer types, including a phase 1 study of tivozanib monotherapy in patients with NSCLC (ClinicalTrials.gov identifier: NCT00826878), a phase 1 study of tivozanib in combination with paclitaxel in patients with advanced or metastatic breast cancer (ClinicalTrials.gov identifier: NCT00717340), and a phase 1 study of tivozanib in combination with FOLFOX6 in patients with advanced colorectal and other gastrointestinal cancers (ClinicalTrials. gov identifier: NCT00660153). 


\section{Axitinib}

Axitinib (AG-013736) is a potent small-molecule inhibitor of all known VEGFRs, with lower potency against PDGFR and c-kit [24]. In a phase 2 study [25•] of 52 patients with metastatic clear cell RCC $(94 \%$ of whom had undergone nephrectomy), axitinib was initiated at $5 \mathrm{mg}$ twice daily. Dose escalation was possible in 6 patients (12\%), and dose reductions were required in $42 \%$ of patients because of grade $2(13 \%)$ and grade $3(29 \%)$ adverse events. Axitinib was associated with an ORR of $44 \%$ ( 2 complete and 21 partial responses), with a median duration of response of 23 months. Median time to progression was 15.7 months, and median OS was 29.9 months; PFS was not reported. Adverse events observed in $>20 \%$ of patients were diarrhea $(60 \%)$, hypertension $(58 \%)$, fatigue $(52 \%)$, nausea $(44 \%)$, dysphonia (37\%), anorexia (35\%), dry skin (33\%), weight loss (27\%), dyspepsia (23\%), and vomiting (21\%). Grade 3 or 4 treatment-related adverse events included hypertension $(15 \%)$, diarrhea $(5 \%)$, and fatigue $(4 \%)$. Hypertension of any grade was reported in $30(58 \%)$ patients but resolved with antihypertensive treatment in all but 8 patients ( 7 of whom had a previous history of hypertension) [25•]. In a second phase 2 study [26•] involving 62 patients with sorafenib-refractory metastatic RCC (95\% with clear cell histology, all had undergone nephrectomy), axitinib $5 \mathrm{mg}$ twice daily provided an ORR of $23 \%$, with a median duration of response of 17.5 months. An additional 21 (34\%) patients had stable disease. Median PFS was 7.4 months, and median OS was 13.6 months. The most common adverse events were fatigue $(77 \%)$, diarrhea (61\%), anorexia (48\%), hypertension (45\%), nausea (44\%), and dyspnea (39\%). Hand-foot syndrome (35\%) and mucositis (34\%) were also common. Grade 3 or 4 adverse events included hand-foot syndrome (16\%), fatigue (16\%), hypertension (16\%), dyspnea (15\%), diarrhea $(15 \%)$, dehydration ( $8 \%$ ), and hypotension (7\%). There appears to be an association between hypertension and efficacy of axitinib: a pooled analysis [27] of phase 2 data demonstrated that median OS for patients with at least one diastolic blood pressure measurement $\geq 90 \mathrm{~mm} \mathrm{Hg}(n=59)$ during axitinib therapy was 130 weeks compared with 42 weeks for patients without elevated diastolic blood pressure $(n=50 ; P<0.01)$. No apparent relationship between drug concentrations and maximum diastolic blood pressure was observed. Axitinib is currently being compared with sorafenib predominantly in the second-line setting in two phase 3 studies in patients with treatment refractory metastatic clear cell RCC (ClinicalTrials. gov identifiers: NCT00678392 and NCT00920816).

Axitinib has also demonstrated efficacy in patients with several other cancer types. As monotherapy, axitinib showed activity against thyroid cancers in a phase 2 study $(n=60)$ [28•], yielding an ORR of $30 \%$ and a median PFS of 18.1 months. In a phase 2 study [29] of 32 patients with stage IV melanoma, treatment with axitinib resulted in an ORR of $16 \%$, a median PFS of 2.3 months, and a median OS of 13.0 months in patients with diastolic blood pressure $\geq 90 \mathrm{~mm} \mathrm{Hg}$ and 6.2 months for those without. In advanced non-small cell lung cancer (NSCLC), a disease control rate of $41 \%$, median PFS of 4.9 months, and median OS of 14.8 months were achieved with axitinib in a phase 2 study $(n=32)\left[30^{\bullet}\right]$.

Axitinib has also demonstrated activity in advanced NSCLC and other solid tumors in combination with chemotherapy in a phase 1 study $(n=47)$ [31]: ORR was $29 \%$ when combined with paclitaxel plus carboplatin (platinum-naive and taxane-naive patients) and $26 \%$ when combined with gemcitabine plus cisplatin (pretreated patients). In a randomized phase 2 study $(n=168)$ [32], axitinib combined with docetaxel showed promising activity in metastatic breast cancer, with a median time to progression of 8.2 months with the combination versus 7 months with docetaxel alone $(P=$ 0.052 ) and an ORR of $40 \%$ with the combination versus $23 \%$ with docetaxel alone $(P=0.038)$. A phase 1 study [33] assessed the combination of axitinib with bevacizumab, a monoclonal antibody to the VEGF ligand, plus chemotherapy (FOLFOX) compared with axitinib plus chemotherapy (FOLFOX or FOLFIRI) in 30 patients with metastatic colorectal cancer and other solid tumors. Responses were observed with all treatment combinations, although patient numbers were too small for statistical comparisons [33]. In contrast to the other cancer types evaluated, the addition of axitinib to gemcitabine in pancreatic cancer has demonstrated only small nonsignificant clinical improvements compared with gemcitabine alone in phase $2(n=103)$ [34] and phase $3(n=632)$ [35] studies, and is not recommended for further evaluation.

Across all cancer types, the most common adverse events observed with axitinib treatment were hypertension, gastrointestinal events, fatigue, anorexia, and hematologic

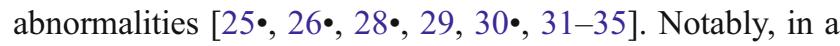
phase 1 study [33] of patients with colorectal and other cancers, the incidence of hypertension was $81 \%$ among patients receiving axitinib plus bevacizumab and chemotherapy versus $27 \%$ among those receiving axitinib plus chemotherapy without bevacizumab.

Several additional clinical studies are ongoing to evaluate axitinib therapy in patients with the above cancers as well as advanced gastric cancers, soft tissue sarcomas, and acute myeloid leukemia or myelodysplastic syndrome (ClinicalTrials.gov).

Cediranib

Cediranib (AZD2171) is an oral VEGFR TKI that has affinity for the VEGFRs, c-kit, PDGFR $\beta$, fibroblast growth 
factor receptor (FGFR)-1, and several other kinases [36]. In a phase 2 study [37], 71 patients with advanced or metastatic RCC were randomized to 12 weeks of treatment with cediranib $45 \mathrm{mg} /$ day $(n=53)$ or placebo $(n=18)$. The mean change in tumor size from baseline was significantly greater among patients randomized to cediranib $(20 \%$ reduction) versus placebo ( $19 \%$ increase; $P<0.0001)$, with partial responses observed in $34 \%$ of patients in the cediranib arm. Median PFS was also significantly greater with cediranib (12.1 months) versus placebo (2.7 months; $P$ $=0.017$ ). Common grade 3 or 4 adverse events included fatigue (19\%), hypertension (19\%), and diarrhea (13\%); 58 $(87 \%)$ patients required a dose reduction or interruption due to toxicities [37]. Preliminary results from another phase 2 study [38] of 43 patients with metastatic RCC have shown partial responses in $38 \%$ of patients and a median PFS of 8.7 months during treatment with cediranib $45 \mathrm{mg} /$ day. Treatment-related grade 3 or 4 adverse events included hypertension $(30 \%)$, fatigue $(26 \%)$, joint pain $(12 \%)$, dyspnea (12\%), and abdominal pain (5\%).

Cediranib monotherapy has also demonstrated promising efficacy in patients with a range of other cancers. In an open-label exploratory study [39] involving 19 patients with recurrent or metastatic head and neck cancer or NSCLC, $6(35 \%)$ patients showed a reduction in tumor metabolic activity of $\geq 25 \%$ (assessed by fluorodeoxyglucose positron emission tomography) after 71 days of treatment with cediranib $30 \mathrm{mg} /$ day. In a phase 2 study [40] of patients with recurrent glioblastoma, treatment with cediranib $45 \mathrm{mg}$ /day resulted in radiographic partial response in $27 \%$ to $57 \%$ of patients, depending upon assessment methodology; the median PFS was 3.8 months, and median OS was 7.5 months. In another phase 2 study [41] involving 47 patients with recurrent epithelial ovarian, fallopian tube, or peritoneal cancer, treatment with cediranib provided clinical benefit (response or stable disease) in $14(30 \%)$ patients; the original dose of cediranib was $45 \mathrm{mg} /$ day, but was subsequently reduced to $30 \mathrm{mg} /$ day because of toxicities in the first 11 patients (primarily fatigue $[52 \%]$ and diarrhea [31\%]). Preliminary results from a phase 2 study [42] in men with castration-resistant prostate cancer that had progressed on docetaxel therapy showed evidence of antitumor activity with cediranib $20 \mathrm{mg} /$ day, with 19 of 34 patients achieving tumor regression, including 6 with partial responses.

Cediranib has also been investigated in a number of combination regimens in breast, colorectal, NSCLC, and small cell lung cancer. Studies of cediranib in combination with chemotherapy in patients with advanced lung cancers have produced inconsistent results, which typically did not demonstrate significant improvement with the addition of cediranib [43-46]. The ORR for patients with NSCLC ranged from $16 \%$ to $38 \%$ with cediranib and $16 \%$ to $18 \%$ without; median PFS ranged from 5.6 to 6.3 months with cediranib to 4.5 to 5.0 months without [43-45]. Further, addition of cediranib was associated with dose reduction/ interruption and/or discontinuation due to toxicity in a majority of patients from each study $[43,44,46]$. Similar results have been observed for cediranib $20 \mathrm{mg}$ /day in combination with FOLFOX chemotherapy versus bevacizumab plus chemotherapy as first-line therapy in patients with metastatic colorectal cancer [47], and for cediranib $45 \mathrm{mg} /$ day in combination with fulvestrant (selective estrogen-receptor antagonist) in women with hormonesensitive metastatic breast cancer [48].

Across cancer types, study results have shown that, although generally effective, cediranib at $45 \mathrm{mg}$ /day was not well tolerated [41, 45, 46, 48], with one study in NSCLC indicating that the lower dose of $30 \mathrm{mg}$ /day cediranib in combination with chemotherapy was not well tolerated either [44]. Overall, the most frequently reported toxicities with cediranib include hematologic abnormalities, fatigue, hypertension, anorexia, dysphonia, gastrointestinal events, and hepatobiliary abnormalities [37-39, 41, 44-46, 48].

Several ongoing clinical trials are evaluating cediranib in patients with the above cancer types as well as in patients

Table 2 Cross trial comparison of selected "off-target" effects and the rates of dose reduction or dose interruptions with VEGFR TKIs in RCC

\begin{tabular}{|c|c|c|c|c|c|}
\hline All grades (grades 3-4) & $\begin{array}{l}\text { Sunitinib [62] } \\
(n=375)\end{array}$ & $\begin{array}{l}\text { Sorafenib [63] } \\
(n=451)\end{array}$ & $\begin{array}{l}\text { Pazopanib }[15 \bullet \bullet] \\
(n=290)\end{array}$ & $\begin{array}{l}\text { Axitinib }[25 \cdot] \\
(n=52)\end{array}$ & $\begin{array}{l}\text { Tivozanib }[20 \bullet] \\
(n=272)\end{array}$ \\
\hline \multicolumn{6}{|l|}{ Adverse events } \\
\hline Mucositis/stomatitis & $43 \%(10 \%)$ & NA & NA & $9 \%(1 \%)$ & $4 \%(<1 \%)$ \\
\hline Hand-foot syndrome & $21 \%(5 \%)$ & $30 \%(6 \%)$ & NA & NA & $4 \%(<1 \%)$ \\
\hline Rash/desquamation & $27 \%(1 \%)$ & $40 \%(1 \%)$ & NA & $6 \%(0 \%)$ & $6 \%(1 \%)$ \\
\hline Fatigue & $58 \%(9 \%)$ & $37 \%(6 \%)$ & $19 \%(2 \%)$ & $27 \%(4 \%)$ & $8 \%(2 \%)$ \\
\hline Diarrhea & $58 \%(6 \%)$ & $43 \%(2 \%)$ & $52 \%(4 \%)$ & $31 \%(5 \%)$ & $12 \%(2 \%)$ \\
\hline Dose reduction & $32 \%$ & $13 \%$ & NA & $29 \%$ & $10 \%$ \\
\hline Dose interruption & $38 \%$ & $21 \%$ & $14 \%$ & NA & $4 \%$ \\
\hline
\end{tabular}

$N A$ not available; $R C C$ renal cell carcinoma; TKI tyrosine kinase inhibitor; VEGFR vascular endothelial growth factor receptor. 
with advanced biliary tract cancers, leukemias, melanoma, and soft tissue sarcomas (ClinicalTrials.gov).

\section{Other TKIs in Development with VEGFR Affinity}

Several other TKIs with anti-VEGFR affinity are also in various stages of clinical development, although most are novel multitargeted TKIs. BIBF 1120 is a potent blocker of VEGFR, PDGFR, and FGFR kinase activity, which has shown antitumor activity and acceptable tolerability in preclinical models [49]. Results from a phase 2 study [50] suggest that maintenance therapy with BIBF 1120 at $250 \mathrm{mg}$ twice daily could delay disease progression in ovarian cancer after previous response to chemotherapy [50]. BMS-690514 is a potent and reversible inhibitor of VEGFR, EGFR, human epidermal growth factor (HER)-2, and HER-4 [51]. In a phase 1 study [51] of 30 patients with a variety of advanced or metastatic solid tumors, BMS-690514 at the maximum tolerated dose of $150 \mathrm{mg} /$ day plus paclitaxel and carboplatin produced partial responses in 9 (30\%) patients. Brivanib (BMS-582664) is a dual inhibitor of VEGFR-2 and FGFR-1 [52] that has shown evidence of activity against hepatocellular cancer in a phase 2 study ( $n=96$ ) [53]. Dovitinib (TKI258), an inhibitor of FGFR, VEGFR, PDGFR, and other tyrosine kinases, has demonstrated clinical activity and acceptable toxicity in preliminary reports from a phase $1 / 2$ study $(n=60)$ in RCC [54] and a phase 1 study $(n=19)$ in melanoma [55]. Motesanib (AMG 706), an inhibitor of VEGF, PDGF, and c-kit receptors, has demonstrated efficacy in combination with paclitaxel and carboplatin similar to that observed with bevacizumab plus chemotherapy in a phase 2 open-label study $(n=181)$ [56] in advanced NSCLC. A phase $1 \mathrm{~b}$ study $(n=26)$ [57] of motesanib demonstrated a good tolerability profile when combined with gemcitabine in the treatment of solid tumors. Vandetanib (ZD6474), a dual inhibitor of VEGFR and EGFR tyrosine kinases, has demonstrated efficacy in NSCLC and medullary thyroid cancer, while negative results have been observed in phase 2 studies in small cell lung cancer, metastatic breast cancer, and multiple myeloma [58]. The feasibility and tolerability of the dual VEGFR and PDGFR inhibitor telatinib (BAY 57-9352) has been demonstrated in a phase 2 study [59] in patients with advanced gastric and gastroesophageal cancers. A phase 1 study [60] in patients with advanced NSCLC $(n=23)$ has demonstrated acceptable tolerability with regorafenib (BAY 73-4506), a multikinase inhibitor of all three VEGFRs, PDGFR, FGFR, c-kit, and several other receptors. Vatalanib (PTK787/ZK222584), an inhibitor of VEGFR-1, -2, and -3 , has shown efficacy in stabilizing metastatic melanoma in a phase 2 study [61]. Studies of the above agents in a variety of cancer types are currently planned or ongoing (ClinicalTrials.gov).

\section{Conclusions}

Currently available multitargeted agents provide important clinical benefits for patients with VEGF-driven tumors, such as RCC. However, these agents are also associated with off-target toxicities that limit their effectiveness. The development of second-generation VEGFR TKIs with improved potency and selectivity has the potential to provide more effective and better tolerated treatment options, enabling rationally designed combination therapies. Available data from clinical studies suggest that second-generation TKIs are generally associated with lower off-target toxicities (Table 2). Ongoing and future studies will further evaluate the clinical effectiveness and tolerability of VEGFR TKIs in a variety of tumor types.

Acknowledgment Editorial support was provided by Kimberly Brooks, PhD, of MedErgy (Yardley, PA) and was funded by AVEO Pharmaceuticals, Inc.

Disclosure Both authors are employees of AVEO Pharmaceuticals, Inc.

Open Access This article is distributed under the terms of the Creative Commons Attribution Noncommercial License which permits any noncommercial use, distribution, and reproduction in any medium, provided the original author(s) and source are credited.

\section{References}

Papers of particular interest, published recently, have been highlighted as:

- Of importance

•- Of major importance

1. Liekens S, De CE, Neyts J: Angiogenesis: regulators and clinical applications. Biochem Pharmacol 2001, 61:253-270.

2. Bergers G, Benjamin LE: Tumorigenesis and the angiogenic switch. Nat Rev Cancer 2003, 3:401-410.

3. Rini BI: Vascular endothelial growth factor-targeted therapy in renal cell carcinoma: current status and future directions. Clin Cancer Res 2007, 13:1098-1106.

4. •Rini BI, Michaelson MD, Rosenberg JE, et al: Antitumor activity and biomarker analysis of sunitinib in patients with bevacizumabrefractory metastatic renal cell carcinoma. J Clin Oncol 2008, 26:3743-3748. This is a phase 2 study demonstrating substantial antitumor activity with sunitinib in patients with bevacizumabrefractory metastatic $R C C$.

5. Valtola R, Salven P, Heikkila P, et al: VEGFR-3 and its ligand VEGF-C are associated with angiogenesis in breast cancer. Am J Pathol 1999, 154:1381-1390. 
6. Jia H, Bagherzadeh A, Bicknell R, et al: Vascular endothelial growth factor (VEGF)-D and VEGF-A differentially regulate KDR-mediated signaling and biological function in vascular endothelial cells. J Biol Chem 2004, 279:36148-36157.

7. Zhang F, Tang Z, Hou X, et al: VEGF-B is dispensable for blood vessel growth but critical for their survival, and VEGF-B targeting inhibits pathological angiogenesis. Proc Natl Acad Sci USA 2009, 106:6152-6157.

8. Rini BI: Metastatic renal cell carcinoma: many treatment options, one patient. J Clin Oncol 2009, 27:3225-3234.

9. Kondo K, Kim WY, Lechpammer M, Kaelin WG, Jr: Inhibition of HIF2a is sufficient to suppress pVHL-defective tumor growth. PLoS Biol 2003, 1:439-444.

10. Maranchie JK, Vasselli JR, Riss J, et al: The contribution of VHL substrate binding and HIF1-alpha to the phenotype of VHL loss in renal cell carcinoma. Cancer Cell 2002, 1:247-255.

11. Ferrara N, Hillan KJ, Gerber HP, Novotny W: Discovery and development of bevacizumab, an anti-VEGF antibody for treating cancer. Nat Rev Drug Discov 2004, 3:391-400.

12. • Escudier B, Eisen T, Stadler WM, et al: Sorafenib in advanced clear-cell renal-cell carcinoma. N Engl J Med 2007, 356:125-134. This phase 3 randomized placebo-controlled study demonstrates prolongation of PFS with sorafenib treatment in advanced clear cell RCC that was resistant to standard therapy.

13. • Motzer RJ, Hutson TE, Tomczak P, et al: Sunitinib versus interferon alfa in metastatic renal-cell carcinoma. N Engl J Med 2007, 356:115-124. This is a phase 3 randomized study demonstrating improved efficacy of sunitinib versus interferon- $\alpha$ against metastatic RCC.

14. Motzer RJ, Michaelson MD, Rosenberg J, et al: Sunitinib efficacy against advanced renal cell carcinoma. J Urol 2007, 178:1883-1887.

15. • Sternberg CN, Davis ID, Mardiak J, et al: Pazopanib in locally advanced or metastatic renal cell carcinoma: results of a randomized phase III trial. J Clin Oncol 2010, 28:1061-1068. This is a phase 3 randomized placebo-controlled study demonstrating improved PFS and tumor response with pazopanib in advanced RCC.

16. Shepard DR, Garcia JA: Toxicity associated with the long-term use of targeted therapies in patients with advanced renal cell carcinoma. Expert Rev Anticancer Ther 2009, 9:795-805.

17. Food and Drug Administration. NDA 22465: VOTRIENT ${ }^{\mathrm{TM}}$ (pazopanib) Tablets. Applicant: GlaxoSmithKline. FDA Presentation ODAC Meeting Oct. 5, 2009. http://www.fda.gov/downloads/ AdvisoryCommittees/CommitteesMeetingMaterials/Drugs/Onco logicDrugsAdvisoryCommittee/UCM186336.pdf. Accessed October 28, 2010.

18. Nakamura K, Taguchi E, Miura T, et al: KRN951, a highly potent inhibitor of vascular endothelial growth factor receptor tyrosine kinases, has antitumor activities and affects functional vascular properties. Cancer Res 2006, 66:9134-9142.

19. Eskens FALM, De Jonge MJA, Esteves B, et al: Updated results from a phase I study of AV-951 (KRN951), a potent and selective VEGFR-1, -2 and -3 tyrosine kinase inhibitor, in patients with advanced solid tumors. Proceedings of the 99th Annual Meeting of the American Association for Cancer Research, San Diego, CA; April 12-16, 2008.

20. •Bhargava P, Esteves B, Al-Adhami M, et al: Activity of tivozanib (AV-951) in patients with renal cell carcinoma (RCC): subgroup analysis from a phase II randomized discontinuation trial (RDT). J Clin Oncol 2010, 28: Abstract 4599. This is a phase 2 study demonstrating antitumor activity and good tolerability of tivozanib in patients with RCC, particularly those with clear cell RCC who had undergone nephrectomy.

21. - Nosov DA, Bhargava P, Esteves B, et al: Phase 2 randomized discontinuation trial (RDT) of tivozanib in patients with renal cell carcinoma (RCC): results in patients randomized to tivozanib vs. placebo [abstract 4906]. Presented at the 35th Annual Congress of the European Society for Medical Oncology, Milan, Italy; October 11-12, 2010. Phase 2 study results demonstrating significant efficacy for patients randomized to tivozanib versus placebo.

22. Bhargava P, Esteves B, Al-Adhami M, et al: Effect of hypertension, nephrectomy and prior treatment on the efficacy of tivozanib (AV-951) in a phase 2 randomized discontinuation trial (RDT) in patients with renal cell carcinoma (RCC) [abstract 342]. Presented at the American Society of Clinical Oncology (ASCO) Genitourinary Cancers Symposium, San Francisco, CA; March 5-7, 2010.

23. Fishman MN, Srinivas S, Hauke RJ, et al: Combination of tivozanib (AV-951) and temsirolimus in patients with renal cell carcinoma (RCC): preliminary results from a phase 1 trial. Mol Cancer Ther 2009, 8: Abstract B60.

24. Rugo HS, Herbst RS, Liu G, et al: Phase I trial of the oral antiangiogenesis agent AG-013736 in patients with advanced solid tumors: pharmacokinetic and clinical results. J Clin Oncol 2005, 23:5474-5483.

25. $\cdot$ Rixe O, Bukowski RM, Michaelson MD, et al: Axitinib treatment in patients with cytokine-refractory metastatic renal-cell cancer: a phase II study. Lancet Oncol 2007, 8:975-984. This phase 2 study shows clinical activity of axitinib in metastatic RCC that did not respond to previous cytokine-based therapy.

26. •Rini BI, Wilding G, Hudes G, et al: Phase II study of axitinib in sorafenib-refractory metastatic renal cell carcinoma. J Clin Oncol 2009, 27:4462-4468. This is a phase 2 study demonstrating antitumor activity of axitinib in metastatic RCC that did not respond to prior VEGF-targeted therapy, including sorafenib.

27. Rixe O, Dutcher J, Motzer R, et al: Diastolic blood pressure (dBP) and pharmacokinetics (PK) as predictors of axitinib efficacy in metastatic renal cell cancer (mRCC). J Clin Oncol 2009, 27: Abstract 5045 .

28. Cohen EE, Rosen LS, Vokes EE, et al: Axitinib is an active treatment for all histologic subtypes of advanced thyroid cancer: results from a phase II study. J Clin Oncol 2008, 26:4708-4713. This is a phase 2 study demonstrating promising activity of axitinib in thyroid cancer.

29. Fruehauf JP, Lutzky J, McDermott DF, et al: Axitinib (AG013736) in patients with metastatic melanoma: a phase II study. J Clin Oncol 2008, 26: Abstract 9006.

30. $\cdot$ Schiller JH, Larson T, Ou SH, et al: Efficacy and safety of axitinib in patients with advanced non-small-cell lung cancer: results from a phase II study. J Clin Oncol 2009, 27:3836-3841. This is a phase 2 study showing antitumor activity and good tolerability of axitinib in advanced NSCLC.

31. Martin LP, Kozloff MF, Krzakowski M, et al: Axtinib (AG013736; AG) combined with chemotherapy in patients (pts) with advanced non-small cell lung cancer (NSCLC) and other solid tumors. J Clin Oncol 2009, 27: Abstract 3559.

32. Rugo HS, Stopeck A, Joy AA, et al: A randomized, double-blind phase II study of the oral tyrosine kinase inhibitor (TKI) axitinib (AG-013736) in combination with docetaxel (DOC) compared to DOC plus placebo (PL) in metastatic breast cancer (MBC). J Clin Oncol 2007, 25: Abstract 1003.

33. Sharma S, Abhyankar V, Burgess RE, et al: A phase I study of axitinib (AG-013736) in combination with bevacizumab plus chemotherapy or chemotherapy alone in patients with metastatic colorectal cancer and other solid tumors. Ann Oncol 2010, 21:297-304.

34. Spano JP, Chodkiewicz C, Maurel J, et al: Efficacy of gemcitabine plus axitinib compared with gemcitabine alone in patients with advanced pancreatic cancer: an open-label randomised phase II study. Lancet 2008, 371:2101-2108.

35. Kindler HL, Ioka T, Richel DJ, et al: A double-blinded, placebocontrolled, randomized, phase III study of axitinib (AG-013736; A) plus gemcitabine $(\mathrm{G})$ vs. $\mathrm{G}$ plus placebo $(\mathrm{P})$ in advanced pancreatic cancer (PC) patients (pts). Eur J Cancer Suppl 2009, 7:361. Abstract $\mathrm{O}-6502$. 
36. Lindsay CR, MacPherson IR, Cassidy J: Current status of cediranib: the rapid development of a novel anti-angiogenic therapy. Future Oncol 2009, 5:421-432.

37. Mulders P, Hawkins R, Nathan P, et al: Final results of a phase II randomised study of cediranib (RECENTINTM) in patients with advanced renal cell carcinoma (RCC). Eur J Cancer Suppl 2009, 7:21. Abstract 49LBA

38. Sridhar SS, Mackenzie MJ, Hotte SJ, et al: Activity of cediranib (AZD2171) in patients (pts) with previously untreated metastatic renal cell cancer (RCC). A phase II trial of the PMH Consortium. J Clin Oncol 2008, 26: Abstract 5047.

39. Saura C, Baselga J, Herbst R, et al: Antitumor activity of cediranib in patients with metastatic or recurrent head and neck cancer (HNC) or recurrent non-small cell lung cancer (NSCLC): An open-label exploratory study. J Clin Oncol 2009, 27: Abstract 6023.

40. Batchelor TT, Duda DG, di TE, et al: Phase II study of cediranib, an oral pan-vascular endothelial growth factor receptor tyrosine kinase inhibitor, in patients with recurrent glioblastoma. J Clin Oncol 2010, 28:2817-2823.

41. Matulonis UA, Berlin S, Ivy P, et al: Cediranib, an oral inhibitor of vascular endothelial growth factor receptor kinases, is an active drug in recurrent epithelial ovarian, fallopian tube, and peritoneal cancer. J Clin Oncol 2009, 27:5601-5606.

42. Adelberg D, Karakunnel JJ, Gulley JL, et al: A phase II study of cediranib in post-docetaxel, castration-resistant prostate cancer (CRPC) [abstract 63]. Presented at the American Society of Clinical Oncology (ASCO) Genitourinary Cancers Symposium, San Francisco, CA; March 5-7, 2010.

43. Gadgeel SM, Wozniak A, Edelman MJ, et al: Cediranib, a VEGF receptor 1,2 , and 3 inhibitor, and pemetrexed in patients (pts) with recurrent non-small cell lung cancer (NSCLC). J Clin Oncol 2009, 27: Abstract e19007.

44. Goss GD, Arnold A, Shepherd FA, et al: Randomized, doubleblind trial of carboplatin and paclitaxel with either daily oral cediranib or placebo in advanced non-small-cell lung cancer: NCIC Clinical Trials Group BR24 study. J Clin Oncol 2010, 28:49-55.

45. Dy GK, Mandrekar SJ, Nelson GD, et al: A randomized phase II study of gemcitabine $(G)$ and carboplatin $(C)$ with or without cediranib (AZD2171 [CED]) as first-line therapy in advanced non-small cell lung cancer (NSCLC). J Clin Oncol (Meeting Abstracts) 2010, 28:7603.

46. Ramalingam SS, Belani CP, Mack PC, et al: Phase II study of cediranib (AZD 2171), an inhibitor of the vascular endothelial growth factor receptor, for second-line therapy of small cell lung cancer. J Thorac Oncol 2010, 5:1279-1284.

47. AstraZeneca. RECENTIN did not meet primary endpoint in Horizon III study in metastatic colorectal cancer. http://www. astrazeneca.com/media/latest-press-releases/2010-new/recoentinhorizon?itemId=8748245. Accessed August 5, 2010.

48. Hyams DM, de Oliveira C, Snyder R, et al: Cediranib in combination with fulvestrant in hormone-sensitive metastatic breast cancer: a phase II randomized study [abstract 204]. Presented at the 32nd Annual San Antonio Breast Cancer Symposium, San Antonio, TX; December 9-13, 2009.

49. Hilberg F, Roth GJ, Krssak M, et al: BIBF 1120: triple angiokinase inhibitor with sustained receptor blockade and good antitumor efficacy. Cancer Res 2008, 68:4774-4782.

50. Ledermann JA, Rustin GJ, Hackshaw A, et al: A randomized phase II placebo-controlled trial using maintenance therapy to evaluate the vascular targeting agent BIBF 1120 following treatment of relapsed ovarian cancer (OC). J Clin Oncol 2009, 27: Abstract 5501

51. Chow LQ, Laurie SA, Belani CP, et al: Phase I trial of BMS690514 in combination with paclitaxel/carboplatin (PC) in patients with advanced or metastatic solid tumors. J Clin Oncol 2010, 28: Abstract 2547.

52. Cai ZW, Zhang Y, Borzilleri RM, et al: Discovery of brivanib alaninate ((S)-((R)-1-(4-(4-fluoro-2-methyl-1H-indol-5-yloxy)-5methylpyrrolo[2,1-f] [1,2,4]triazin-6-yloxy)propan-2-yl)2-aminopropanoate), a novel prodrug of dual vascular endothelial growth factor receptor-2 and fibroblast growth factor receptor-1 kinase inhibitor (BMS-540215). J Med Chem 2008, 51:1976-1980.

53. Raoul JL, Finn RS, Kang YK, et al: An open-label phase II study of first- and second-line treatment with brivanib in patients with hepatocellular carcinoma (HCC). J Clin Oncol 2009, 27: Abstract 4577.

54. Angevin E, Grunwald V, Lin C-C, et al: A phase I/II study of TKI258 (dovitinib), a FGFR and VEGFR inhibitor, in patients (pts) with advanced or metastatic renal cell cancer (mRCC): preliminary phase II results. Ann Oncol 2010, 21: Abstract 507P.

55. Kim KB, Saro J, Moschos SS, et al: A phase I dose finding and biomarker study of TKI258 (dovitinib lactate) in patients with advanced melanoma. J Clin Oncol 2008, 26: Abstract 9026.

56. Blumenschein GR, Kabbinavar FF, Menon H, et al: Randomized, open-label phase II study of motesanib or bevacizumab in combination with paclitaxel and carboplatin $(\mathrm{P} / \mathrm{C})$ for advanced nonsquamous non-small cell lung cancer (NSCLC). J Clin Oncol 2010, 28:7528.

57. Price TJ, Lipton L, McGreivy J, et al: Safety and pharmacokinetics of motesanib in combination with gemcitabine for the treatment of patients with solid tumours. Br J Cancer 2008, 99:1387-1394.

58. Morabito A, Piccirillo MC, Falasconi F, et al: Vandetanib (ZD6474), a dual inhibitor of vascular endothelial growth factor receptor (VEGFR) and epidermal growth factor receptor (EGFR) tyrosine kinases: current status and future directions. Oncologist 2009, 14:378-390

59. Ko AH, Tabernero J, Garcia De Paredes M, et al: Phase II study of telatinib (T) in combination with capecitabine $(\mathrm{X})$ and cisplatin $(\mathrm{P})$ as first-line treatment in patients (pts) with advanced cancer of the stomach $(\mathrm{G})$ or gastro-esophageal junction (GEJ). J Clin Oncol 2010, 28: Abstract e14575.

60. Kies MS, Blumenschein GR, Jr., Christensen O, et al: Phase I study of regorafenib (BAY 73-4506), an inhibitor of oncogenic and angiogenic kinases, administered continuously in patients (pts) with advanced refractory non-small cell lung cancer (NSCLC). J Clin Oncol 2010, 28: Abstract 7585.

61. Cook N, Basu B, Biswas S, et al: A phase 2 study of vatalanib in metastatic melanoma patients. Eur J Cancer 2010, 46:2671-2673.

62. SUTENT ${ }^{\circledast}$ (sunitinib malate) capsules, oral [package insert]. New York, NY: Pfizer Inc, 2010.

63. NEXAVAR (sorafenib) tablets, oral [package insert]. Wayne, NJ: Bayer HealthCare Pharmaceuticals Inc, 2009.

64. Chow LQ, Eckhardt SG: Sunitinib: from rational design to clinical efficacy. J Clin Oncol 2007, 25:884-896.

65. Ratain MJ, Eisen T, Stadler WM, et al: Phase II placebocontrolled randomized discontinuation trial of sorafenib in patients with metastatic renal cell carcinoma. J Clin Oncol 2006, 24:2505-2512.

66. Sloan B, Scheinfeld NS: Pazopanib, a VEGF receptor tyrosine kinase inhibitor for cancer therapy. Curr Opin Investig Drugs 2008, 9:1324-1335. 